SANTAOlalla Montoya, C., «La Ley i2/20I3 de la cadena alimentaria, ¿réplica o complemento de la Ley de defensa de la competencia?», REDUR I4, diciembre 20I6, págs. I37-I60. ISSN I695-078X

\title{
LA LEY I2/2OI3 DE LA CADENA ALIMENTARIA, ¿RÉPLICA O COMPLEMENTO DE LA LEY DE DEFENSA DE LA COMPETENCIA?
}

\author{
Cayetana SANTAOLALLA MONTOYA \\ INVESTIGADORA PRE-DOCTORAL EN DERECHO INTERNACIONAL PRIVADO \\ UNIVERSIDAD DEL PAÍS VASCO
}

SumARIO: I. Consideraciones introductorias. II. Antecedentes históricos de la Ley de la cadena alimentaria y su contenido material. III. Legislación de defensa de la competencia y la Ley de la cadena alimentaria. IV. Conclusiones.

RESUMEN: La Ley I2/2013 de medidas para mejorar el funcionamiento de la cadena alimentaria entró en vigor el i de enero de 20I4. Su objetivo era proteger al sector primario como escalón más débil dentro de la cadena alimentaria y darle más fuerza en las negociaciones con los compradores y los mayoristas. Su aprobación y el funcionamiento a través de los inspectores de la Agencia de Información y Control Alimentario (AICA) cuestiona la labor que no ha realizado la legislación de defensa de la competencia con el sector agroalimentario. Este artículo muestra el origen, la evolución y las razones que han sido necesarias para que se aprobara esta ley especial. Y aclara el interrogante de si la Ley I2/20I3 complementa a la actual legislación de defensa de la competencia o si representa una duplicidad de esfuerzos económicos, materiales y humanos.

Palabras Clave: Ley I2/20I3, cadena alimentaria, defensa de competencia, sector primario, agroalimentario, AICA

ABSTRACT: Law I2/20I3 of measures for improving the functioning of the food supply chain entered into force on Ist January 20I4. Its main objective was protecting the primary sector like the weakest link at food supply channel and giving greater strength in the negotiations with buyers and distributors. When this law was approved and started to run through inspectors by food control and information Agency, the ineffectiveness of competence law regarding agrifood sector was questioned. This paper exposes the origin, arguments and evolution of this special law in order to answer an important matter: does this law of food supply chain in Spain duplicate or complete the competence law in force?

KEYWORDS: Law I2/20I3, food chain, preservation of competition, primary sector, agrifood, AICA 


\section{Consideraciones introductorias}

Es habitual leer en la prensa española noticias que entremezclan conceptos de la ley de defensa de la competencia, la ley de la cadena alimentaria y la nueva Agencia de Información y Control alimentarios (AICA) enmarañando los conceptos porque no están claros. Se genera más confusión al lector de la que ya tiene. Voy a poner unos ejemplos de que lo que ocurre: «Petición a la AICA para que investigue los bajos precios de la leche en Galicia» ${ }^{1}$ porque existe una infracción de la Ley I5/2007 de defensa de la competencia, concretamente de su artículo 62. «Se solicita a la AICA una investigación por presunto caso de venta a pérdidas en aceite de oliva» ${ }^{2}$. Estas noticias abundan cada vez más en la prensa nacional, económica y agraria especializada. Y si no fuera porque la AICA no es competente para resolver estos asuntos, no pasaría nada.

Estos titulares llevan a pensar que la Ley I2/20I3 (BOE 3 agosto de 2013) de medidas para mejorar el funcionamiento de la cadena alimentaria (LCA) ha creado, cuando menos, un debate social sobre unos temas delicados que antes también existían pero que la gente y/o las instituciones no sabían ni siquiera que representaban una infracción ni qué autoridad era la encargada de perseguirlos. Otra conclusión que extraigo es que el conocimiento de la legislación de competencia sigue siendo insuficiente, desolador incluso.

Cuando existe una vulneración de la libre competencia en el mercado, todo interesado o afectado debe dirigirse a la autoridad de defensa de la competencia correspondiente en su caso, bien de ámbito autonómico si la hubiera, de ámbito nacional a través de la Comisión Nacional de los Mercados y la Competencia (CNMC) en Madrid o bien a través de la Dirección General de Competencia (DGCompetencia) en la Comisión Europea. De las infracciones en competencia relacionadas con el sector agrícola será competente la Dirección General de Agricultura de la Comisión y no a la DGCompetencia. Es decir, el sector agrícola a nivel europeo recibe un tratamiento diferenciado que destaca sobre el resto de sectores económicos porque ellos serán desviados a Competencia y no a Agricultura.

Por su parte, cuando se producen las ventas a pérdida (o ventas a resultas) ${ }^{3}$, la AICA no es el organismo competente para perseguir, inspeccionar y sancionar estas conductas. Serán los organismos autonómicos correspondientes los encargados de hacerlo porque se trata de una competencia que se ha transferido a las propias CCAA. Corresponderá al Departamento de Agricultura, Desarrollo Económico, Comercio u otro Departamento, según como se haya dispuesto en su propio Estatuto de Autonomía.

Campo Galego, 9.05.20I6, (disponible en http://www.campogalego.com/es/leche/peticion-a-la-aica-paraque-investigue-los-bajos-precios-de-la-leche-en-galicia; fecha de consulta: I5.I0.20I6).

${ }^{2}$ Europapress, I0.06.20I6, (disponible en

http://www.europapress.es/economia/noticia-coag-solicita-aica-investigacion-dia-presunto-caso-venta-

perdidas-aceite-oliva-20I606IoI04950.html; fecha de consulta: 5.I0.20I6).

${ }^{3}$ Artículo $17.2^{\circ}$ de la Ley 3/199I de Competencia Desleal la describe como la venta realizada bajo coste o bajo precio de adquisición. 
Por lo tanto no es de extrañar la confusión que impera entre la población que, cuando sufre una infracción de algún tipo y por fin la identifica (tarea no siempre fácil como se observa), no tiene ni la más remota idea de a quién debe dirigirse para salvaguardar sus derechos. Sin ánimo de desalentar, considero que la AICA está haciendo un gran trabajo, no puedo decir lo mismo de otros organismos con labor inspectora y sancionadora también. Está claro que aún queda mucho trabajo por hacer.

La diferencia de precios entre lo que cobra el agricultor/ganadero y lo que paga el consumidor en el punto de venta sigue siendo uno de los aspectos no resueltos por la Ley de la cadena alimentaria. Esta realidad se extiende a toda la Unión Europea (UE). En la LCA no existe ninguna medida que permita actuar contra el abuso en los márgenes comerciales. Para Vidal Maté, esta ley ha supuesto el reconocimiento de determinados abusos pero no la corrección de los mismos ${ }^{4}$. Opinión que comparto.

Se acuña la expresión de la doble presión inversa $a^{5}$ cuando se alude a la debilidad negociadora de los productores cuando se enfrentan, por un lado, a un proceso de compra de insumos que son cada vez más caros y, por otro lado, al proceso de venta de sus productos a compañías de gran dimensión y poder que cada vez estrechan más sus márgenes.

La Unión de Uniones de agricultores denunció que, mientras los precios que cobra el agricultor bajaron un 3,2\% de media en el último trienio, de 2012 a 2015 , los precios que paga el consumidor por su alimentación registraron un incremento del 3,1\%. Esta situación que se produce en el sector agrario, la industria alimentaria o la distribución española, se repite como un calco en el conjunto de la $\mathrm{UE}^{6}$.

Las prácticas comerciales desleales suelen ser impuestas por una parte que se halla en situación de superioridad frente a otra que se halla en situación de inferioridad, la cual a menudo no está en posición de abandonar la relación desleal y buscar otro socio comercial debido a los costes que implica tal cambio o a su falta de alternativas. La Comisión recogió estas preocupaciones en el Libro Verde sobre prácticas comerciales desleales en la cadena de suministro alimentario y no alimentario entre empresas en Europa ${ }^{7}$ en 2013. La mayoría de las veces se trata de productos frescos, perecederos y los agricultores no disponen de almacenaje por lo que el temor a que nadie les recoja su cosecha o que madure excesivamente en el campo es real. Esa realidad les conduce a aceptar condiciones desiguales en detrimento de sus propios intereses.

El argumento para defender las subvenciones agrarias en la última reforma de la Política Agraria Común (PAC) en 2013 (período 20I4-2020) es disponer de un sector agrario fuerte y competitivo por lo que es conveniente tener una seguridad alimentaria al

\footnotetext{
${ }^{4}$ MATE, V., «Atrapados en la cadena», periódico Noticias de La Rioja, Logroño, 4.05.20I6, pág. I5.

5 LAMO DE ESPINOSA, J., «La crisis económica global y la crisis agraria española», en El futuro de la economía española, Revista Mediterráneo Económico n I6, Almería, 2009, págs.47-83, especialmente págs. 65-68.

${ }^{6}$ MATE, V., op. cit., pág. I5.

${ }^{7}$ Comisión Europea, Bruselas, 3I.I.20I3 COM (20I3) 37 final.
} 
margen de los vaivenes de las producciones mundiales, tanto en volumen como en calidad de la oferta, todo ello a unos precios adecuados a la capacidad adquisitiva de los consumidores. Se habla de una seguridad alimentaria que está poniendo en jaque al mismo Tratado Transatlántico de Comercio e Inversiones (TTIP por sus siglas en inglés) entre Estados Unidos y la UE. El posicionamiento de la Comisión respecto a la seguridad alimentaria que ha sido nuestro estandarte durante años parece claro, se está realizando una defensa a ultranza del mismo. Los temas que generan inquina son las contrataciones públicas y las denominaciones de origen. Por su parte, aspectos no resueltos que conducen a que finalice el actual mandato americano sin finalizar ni firmar este acuerdo comercial bilateral que tantas expectativas ha generado y tantas manifestaciones públicas de rechazo también.

Parece claro que contraviene el mismo espíritu de la PAC el hecho de que se mantengan esos fuertes desequilibrios entre los precios que percibe el productor y los que paga finalmente el consumidor. El campo hace un esfuerzo por producir más barato y no se nota en la cesta de la compra porque hay una subida excesiva de precios que se produce en el camino. El ciudadano se pregunta para qué sirven las ayudas agrarias pagadas con sus impuestos si paga una alimentación más cara por esa cadena mal equilibrada. Desde las organizaciones agrarias, se pide al Parlamento Europeo que analice la situación que perjudica a los productores y a los consumidores en beneficio solo de un pequeño eslabón dominante de la cadena. Opinión que comparto plenamente. De qué sirve proteger durante años a nuestro sector agrícola para hacerlo competitivo, sostenible y generar empleo a través de él, si se permiten unos desequilibrios tan grandes en la cadena alimentaria que favorecen solo a unos pocos.

La aprobación de la Ley de la cadena alimentaria responde a varias inquietudes que alcanzan su punto álgido en 20I3. En primer lugar, las sucesivas reformas de la PAC ponen de manifiesto que es necesario adoptar medidas para fortalecer el poder de negociación de los agricultores como eslabón más débil en la cadena alimentaria. Aparece como un imperativo que se debe atender a pesar de que la reforma de la PAC de 2013 fue criticada por generalista e inconclusa, por no dar respuesta a los problemas reales del sector. Es en ella donde surge la necesidad de abordar las normas de competencia en su relación con la PAC. Las instituciones comunitarias exponen abiertamente su preocupación ${ }^{8}$. Es cierto que existen estudios que analizan las resoluciones en competencia que vinculan al sector agroalimentario, pero son escasos y apenas concluyentes ${ }^{9}$. Esos estudios preliminares no llegan a resolver los problemas estructurales que tiene el sector.

\footnotetext{
${ }^{8}$ Dictamen del CESE sobre relaciones comerciales entre la gran distribución $y$ proveedores de productos alimentarios que aparece en el DOUE $\mathrm{CI}_{33}$ de 9 de mayo de 20I3; Libro Verde sobre prácticas comerciales desleales en la cadena de suministro alimentario y no alimentario entre empresas en Europa en 20I3, Bruselas, 3I.I.20I3 COM (2013) 37 final.

${ }^{9}$ Los realizados por la autora Patricia LAMO DE EsPINOSA.
} 
La Comunicación de la Comisión 2009/59 $\mathrm{I}^{\mathrm{IO}}$ insta a los Estados Miembros a mejorar el funcionamiento de la cadena alimentaria a nivel estatal. La Comisión Europea concedió a los Estados miembros prerrogativas para regular la cadena alimentaria a nivel nacional. España se convierte en un referente europeo con la aprobación de su Ley I2/2013. A través del Real Decreto 227/20I4, de 4 de abril, se aprobó el Estatuto de la AICA (BOE 30.04.20I4). Lo más importante del Real Decreto 227/20I4 es que establece que la AICA podrá iniciar de oficio o a instancia de parte los procedimientos y en su caso, comunicar al MAGRAMA o bien a la CNMC para que continúen con el procedimiento en aquellos casos en los que no sea competente. Se debe evitar que un mismo asunto quede enjuiciado por dos vías distintas.

El objetivo de la LCA es mejorar el funcionamiento de la cadena alimentaria en las compraventas de alimentos y materias primas entre agricultores y ganaderos, fabricantes de alimentos y bebidas y distribuidores del sector agroalimentario. Algunos autores consideran que esta ley pretende ajustar la conducta de las grandes superficies que abusan de su situación de poder. Los más favorecidos por la aplicación de esta ley resultarían los agricultores y las empresas transformadoras.

La LCA en España es el resultado del debate producido en el seno de la Comisión Nacional de Competencia ${ }^{\text {II }}$ (CNC) a raíz del Informe sobre las relaciones entre los fabricantes $y$ distribuidores en el sector agroalimentario en $2 \mathrm{OII}^{\mathrm{I2}}$. La CNC considera que el excesivo intervencionismo público del texto legislativo puede constituir un riesgo para la libre competencia ${ }^{\text {I3 }}$. No obstante, la aplicación de la LCA no se ha confiado a las autoridades de defensa de la competencia. Su aplicación se encomienda a organismos y a instituciones administrativas especiales dependientes del Ministerio de Agricultura, Alimentación y Medioambiente (MAGRAMA) y no a las autoridades de competencia.

La LCA busca complementar el ordenamiento de defensa de la competencia. El artículo 7 de la LCA establece que el contenido de las relaciones reguladas por la presente Ley, así como la aplicabilidad de los principios rectores en la ejecución e interpretación de tales relaciones, quedará sometido a la normativa de defensa de la competencia, sin perjuicio de lo dispuesto en la normativa comunitaria.

El derecho de la competencia está sectorializado y se centra en el sector energético, las telecomunicaciones, el audiovisual, el transporte y el postal ${ }^{14}$. El sector agroalimentario

\footnotetext{
${ }^{10}$ Dictamen del Comité Económico y Social Europeo sobre la Comunicación de la Comisión al Parlamento Europeo, al Consejo, al Comité Económico y Social Europeo y al Comité de las Regiones sobre mejorar el funcionamiento de la cadena alimentaria en Europa, DOUE 15.02.20II.

${ }^{\text {II }}$ Comisión Nacional de Competencia (CNC) que pasó a llamarse Comisión Nacional del Mercado de la Competencia (CNMC) con la aprobación de la Ley 3/20I3, de 4 junio (BOE 5.06.20I3), qué creó la CNMC.

${ }^{12}$ CNC, Trabajando por la Competencia, Informe sobre las relaciones entre los fabricantes y distribuidores en el sector alimentario, 20II, Madrid, págs. I-I74.

${ }^{13}$ Moya LATORRe, A. y GUILlÉn FerRer, M.J., Impacto del nuevo proyecto de ley para mejorar el funcionamiento de la cadena alimentaria, Cuatrecasas Goncalves Pereira, Barcelona, 2013, págs. 663-676, especialmente pág. 666.

${ }^{14}$ División departamental de la CNMC, (disponible en https://www.cnmc.es/; fecha de consulta: O2.0I.20I6)
} 
apenas se menciona ni tiene asignada dirección específica dentro del órgano nacional, la CNMC. Las distintas autoridades de competencia afirman que al sector agroalimentario se le aplican todas las reglas de competencia al igual que al resto de sectores, pero en la práctica aparece como un sector residual apenas abordado por las resoluciones de las autoridades de competencia.

La doctrina considera que es precisamente debido a este motivo por el que surge la necesidad de aprobar la Ley I2/20I3 de medidas para mejorar el funcionamiento de la cadena alimentaria. Esta ley supondría la evidencia de un trabajo que no han hecho las autoridades de competencia. Voy a defender la tesis de que esta ley surge precisamente por el abandono que sufre el sector agroalimentario frente a la legislación de competencia. Se le aplican las leyes de competencia, pero apenas se presta atención a este sector agrícola cuyo valor de producción total en la UE alcanzó en el año 2015 los 4IO millones de euros ${ }^{15}$.

Se considera que la LCA ha aprobado unas normas de intervención extraordinaria a favor de un sector de actividad. Los poderes públicos defienden la parte más débil de la relación. De hecho, existe la presunción de que la multa se impondrá sobre la parte fuerte de la relación contractual. Se trata de disposiciones de defensa de la competencia en sentido material pero divergen en sus técnicas de aplicación. Su aplicación se encomienda a organismos y estructuras específicas a las que se habilita a definir una política de forma análoga a lo que contemplan las normas de defensa de la competencia sin perjuicio del control que lleven a cabo los tribunales de justicia.

Una investigación realizada por el Centro de Investigación en Economía y Desarrollo Agroalimentario (CREDA), a petición de la $\mathrm{AICA}^{16}$, ha demostrado que España es pionera en la regulación de las relaciones en la cadena alimentaria con la aprobación de la LCA y de su organismo AICA dentro del panorama europeo respecto al resto de países miembros. En primer lugar, ha sido uno de los primeros países en implementar una Regulación Específica de la Cadena Alimentaria con cobertura en todos sus eslabones, no solo en los últimos escalones como ocurre en otros Estados miembros como es el caso de Reino Unido, Eslovaquia y República Checa ${ }^{\mathrm{r}}$. Es decir, ha aprobado una legislación específica que se aplica a todo el canal de venta y comercialización del sector agroalimentario. Otros Estados miembros que también lo han hecho han sido Francia, Alemania, Portugal e Italia. En otros Estados, sin embargo, las facultades de regulación de la cadena se han asignado a las respectivas autoridades nacionales de competencia (Alemania, Austria, Bulgaria, Chipre, Hungría, Irlanda, Italia, Letonia, Lituania, República Checa y Rumanía $)^{18}$ y son aplicables a todos los sectores económicos por igual, no exclusivamente al agroalimentario como sí hace la legislación española.

\footnotetext{
${ }^{15}$ COMISIÓN EUROPEA, Informe anual sobre el comercio agroalimentario en 2015: La UE, primer exportador mundial, Bruselas, 20I6, pág.I.

${ }^{16}$ CREDA y AICA, Informe sobre la aplicación de regulaciones de las prácticas comerciales en la cadena alimentaria en los países de la UE, Madrid, 20I6, págs. I- I4O.

${ }^{17}$ Ibidem, pág. 48.

${ }^{18}$ CREDA y AICA, op. cit., pág. 28.
} 
La instrucción de los casos y la supervisión del cumplimiento de la LCA en España lo realiza la AICA que sustituye a la Agencia para el Aceite de Oliva que ha quedado suprimida. La AICA se crea en la Disposición Adicional Primera de la Ley I2 $/ 2013^{\text {19 }}$ y es un organismo específico de control, dependiente del MAGRAMA y opera como sistema público de garantía del cumplimento de la normativa, con plenas capacidades para actuar tanto de oficio como por denuncia de un interesado. Realiza inspecciones, controla operaciones comerciales, recibe denuncias y propone sanciones. Se trata de un organismo autónomo al que la Ley dota de poderes similares a los que tiene la autoridad de competencia. Puede acceder por sorpresa a los locales y terrenos de las empresas, verificar almacenes, acceder a toda la documentación relativa a la actividad de la entidad y precintar instalaciones. Esta es una razón más que avala la tesis que defiendo.

La realidad demuestra, sin embargo, que todavía no hay confianza en que se preserve el anonimato de las denuncias que se pueden presentar ante la AICA, a pesar de que los propios inspectores aseguran que su deber es ese. En concreto, los agricultores son reacios a interponer denuncias. Creen que su identidad se acabará filtrando y pondrá en peligro su propio negocio y el sustento de su familia ${ }^{20}$. El desempeño de la AICA con respecto a otras autoridades de control a nivel europeo destaca por ser el organismo más activo en el control de las prácticas comerciales desleales en la cadena alimentaria en cuanto al número de inspecciones realizadas, operaciones comerciales controladas, denuncias recibidas, expedientes sancionadores abiertos, número de sanciones propuestas y el número de jornadas divulgadas ${ }^{2 \mathrm{I}}$.

Las multas y sanciones en la normativa española se encuentran dentro de las más altas de la UE, a pesar de que haya detractores que destacan que el incremento de la multa se produce por la reincidencia de la conducta y no por otro motivo. Complementariamente a la normativa, implementa instrumentos auto-regulatorios a través del Código de Buenas Prácticas Mercantiles en la Contratación Alimentaria (CBPCA) donde los operadores económicos se comprometen a aplicar de forma voluntaria prácticas mercantiles que fomentan las relaciones justas, equilibradas y leales, lo que caracteriza al caso español como un modelo mixto de regulación ${ }^{22}$.

La legislación nacional española ya tiene los mecanismos que necesita para perseguir las conductas que se detallan en los informes de las instituciones europeas y de los organismos nacionales. Esta es una parte importante del problema. Esos mecanismos aparecen dispersos entre la legislación de defensa de la competencia, la ley de competencia desleal, la ley de patentes y marcas, la legislación de ordenación del comercio minorista y la

\footnotetext{
${ }^{19}$ En el Real Decreto 227/20I4, de 4 de abril, se aprueba el Estatuto de la Agencia de Información y Control Alimentarios.

${ }^{20}$ Encuesta realizada por la autora en febrero de 2015 a los agricultores riojanos.

${ }^{21}$ CREDA y AICA, op.cit., pág. I28.

${ }^{22}$ Ibidem, pág. I27
} 
lucha contra la morosidad ${ }^{23}$. Es decir, la ley en España ya tiene los mecanismos necesarios para perseguir, sancionar y corregir estas conductas que regula la LCA y, sin embargo, no lo hace porque en el sector primario resulta muy difícil denunciar, probar e iniciar los procedimientos.

Esta realidad y esta imposibilidad subyace en el hecho de que se deba aprobar una nueva ley porque las leyes citadas no se están aplicando adecuadamente ¿Se estaría produciendo una duplicidad? La aprobación de la Ley de la cadena alimentaria pone en evidencia las cosas que, por acción u omisión, se han hecho mal y sería conveniente realizar una lectura crítica de lo ocurrido, a pesar de que haya autores que intenten justificar razonadamente esta ley.

No obstante, la LCA adolece de ciertos fallos que han sido criticados por distintos grupos de interés ${ }^{24}$. En primer lugar, no concreta los instrumentos de sanción. El Real Decreto 64/2015, de 6 de febrero (BOE 7.02.2015) establece disposiciones relativas a las buenas prácticas en la contratación alimentaria, al Observatorio de la Cadena Alimentaria y a los laboratorios agroalimentarios para el control oficial, pero no concreta los instrumentos de sanción. En la propia Ley se establecen multas de carácter económico, de cuantía notablemente inferior a las que establece la ley de competencia, pero nada más. Esta inoperatividad la han puesto de relieve sobretodo los sindicatos agrarios.

Es cierto que respecto a la regulación de la cadena alimentaria a nivel europeo, España está muy bien valorada, entre otros motivos, porque sus multas son las más altas del panorama de la UE. Sin embargo, si comparamos las multas económicas de la AICA con las de los organismos nacionales y autonómicos (en los casos que tengan la facultad de imponer sanciones) de defensa de la competencia, está claro que obtendrían una ventaja significativa los infractores de la LCA frente a los infractores de la Ley $15 / 2007$, de 3 de julio, de defensa de la competencia ${ }^{25}$ (LDC) y de la Ley 3/199I, de Io de enero, de competencia desleal $^{26}$ (LCD). Sería conveniente analizar caso por caso y el por qué.

Es bastante común encontrar situaciones en las que se infringen varias leyes a la vez. Es decir, son conductas en las que se pactan precios, se divide el mercado, no hay contratos escritos, se paga al proveedor en un promedio temporal de cinco meses la venta de productos perecederos (en vez de en 30 días como exige la Ley contra la morosidad) y en

\footnotetext{
${ }^{23}$ CRespo, D. y LiÑán, P., Todo lo que usted debe saber sobre la Ley de la Cadena Alimentaria, CMS Albiñana \&Suárez de Lezo, Madrid, 20I4, págs. I-II, especialmente pág. 2.

${ }^{24}$ Sindicato Unións Agrarias, I0.02.2015, (disponible en

http://www.europapress.es/galicia/agro-00246/noticia-rural-uuaa-critica-nuevo-decreto-ley-cadenaalimentaria-no-impide-abusos-industria-lactea-20150210I94559.html; fecha de consulta 02.09.20I6);

Asaja, I0.09.20I4, (disponible en

http://www.finanzas.com/noticias/empresas/20I409ı//asaja-critica-cadena-alimentaria-2752078.html; fecha de consulta I2.09.20I6); Confederación Nacional de Empresarios Mayoristas de Frutas y Hortalizas de España (Coemfe), 4.02.2016, (disponible en http://www.revistaaral.com/es/notices/2016/O2/coemfe-criticaa-agricultura-por-ignorarle-en-la-ley-de-mejora-de-la-cadena-alimentaria-7607I.php\#.V9oitk2LTIU;

fecha de consulta: I5.09.20I6).

${ }^{25}$ Ley I5/2007, de 3 de julio, de defensa de la competencia, BOE 4.07.2007.

${ }^{26}$ Ley 3/I99I, de io de enero, de competencia desleal, BOE II.oI.I99I.
} 
donde existen conductas desleales. Se viven situaciones complejas y lo peor es la falta de información y concienciación sobre la asfixia que todas estas actuaciones juntas representan para los agricultores y sus familias. La persecución y sanción de todas estas actuaciones no es sencilla.

La segunda lectura crítica de la LCA es relativa a la adhesión voluntaria a un Código de Buenas Prácticas Mercantiles que no vincula en absoluto a aquellos que no se hayan adherido al mismo. Tampoco ayuda que se coordine por el MAGRAMA porque el Ministerio no es parte implicada y solo puede asesorar ciñéndose a la ley en vigor. Sin embargo, la norma contempla determinadas medidas que podrá adoptar el MAGRAMA para promover su uso, como la publicidad de las empresas adheridas (recogidas en un Registro Estatal que se crea a estos efectos), la posibilidad que se dará a los operadores de utilizar el correspondiente sello, la realización de campañas de promoción estatal y, sobre todo, la consideración de la adhesión o no al Código como un criterio adicional a los efectos de conceder ayudas y subvenciones.

Estas medidas del MAGRAMA, si se aprueban, están muy bien porque van a incentivar la adherencia al código y la promoción de buenas prácticas en la cadena alimentaria $^{27}$. Sin embargo, es cierto que la adhesión al código de buenas prácticas es voluntaria y eso implica que el que opta libremente por adherirse, luego debe acatar la normativa y la sanción que se le aplique. No se puede uno adherir para lo bueno y no para lo malo. Por otro lado, la adhesión al código no debe ser un requisito puntuable para la obtención de ayudas o subvenciones.

Las ayudas o subvenciones se conceden con objeto de subsanar o enmendar fallos que presenta el mercado, no como forma de premiar o castigar por una determinada actuación. Resultarían injustificadas y discriminatorias. La $\mathrm{CNMC}^{28}$ critica que se concedan ayudas económicas sujetas a la adhesión voluntaria a ese código de buenas prácticas. Las ayudas deben tener por objeto estabilizar los mercados y promover la libre competencia, no favorecer a unos frente a otros por una actuación que no es susceptible de subvención en sí misma.

Con objeto de concretar el Código de buenas prácticas, se deben aclarar dos conceptos que son discutidos en la doctrina y que aparecen como problemas latentes en dicha autorregulación voluntaria. Por un lado, las ventas a pérdida (también llamadas ventas a resultas) y por otro lado, el uso y gestión de las marcas del distribuidor frente a la marca privada. La doctrina considera que ambos problemas no deben ser regulados en la LCA. La CNC considera que la venta a pérdida no debe seguir regulándose en el artículo I4 de la Ley de Ordenación del Comercio Minorista ${ }^{29}$ (LORCOMIN) porque esa figura ya aparece en la Ley de competencia desleal (artículo $17.2^{\circ}$ de la Ley 3/I99I). Mantener ese tipo en la

\footnotetext{
${ }^{27}$ Crespo, D. et al., op.cit., pág. 9.

${ }^{28}$ En el Proyecto IPN/DP/O009/I4 del RD por el que se aprueba el Reglamento de la Ley I2/20I3, de 2 de agosto, de medidas para mejorar el funcionamiento de la cadena alimentaria, CNMC, Madrid, 20I4, págs. I-II.

${ }^{29}$ Ley 7/1996, de I5 de enero, de ordenación del comercio minorista, BOE I7.0I.I996.
} 
LORCOMIN, en opinión de la CNC, es inapropiado y redundante ${ }^{30}$. Hallamos varios supuestos de duplicidad de regulaciones para una misma conducta. Eso nos debería llevar a hacer un análisis de por qué estamos cayendo en esa duplicidad que es un desgaste de recursos económicos, materiales y humanos.

El 24 de noviembre de 2015 se aprobó el CBPCA, de aplicación en todo el territorio español. El Código se aprobó con el acuerdo del MAGRAMA y las organizaciones y asociaciones de ámbito superior al de una Comunidad Autónoma, representativas de los operadores de la producción, la industria o la distribución alimentaria. No obstante, se critica que para la elaboración del mismo se haya prescindido de los mercas $^{31}$ que representan un $40 \%$ de las ventas del sector agroalimentario en el país. Hubiera sido adecuado dialogar con los intermediarios que operan en los mercados centrales de abastecimiento y con las asociaciones de minoristas porque son los que más volumen de operaciones mueven dentro del sector agroalimentario en el país ${ }^{32}$.

El fin último de la LCA es proteger y favorecer al consumidor, en este punto coincide con la legislación de competencia. Otro aspecto que fortalece la tesis que defiendo. Ninguna de las dos leyes tiene como fin último la protección del proveedor/productor/agricultor. Su fin último es la defensa del consumidor final y para ello, promueven la libre competencia en el mercado porque es una manera de garantizar la protección más eficaz de los consumidores. Pienso en la Ley Sherman que fue el origen de las leyes de competencia y observo cómo la protección a los agricultores, con el paso de los años y las adaptaciones que han sufrido las leyes de competencia en el mundo, se ha desvanecido por completo.

\section{Antecedentes históricos de la Ley de la cadena alimentaria y su contenido material}

La subida de los precios agrícolas en 2008 provocó que la atención recayera en el funcionamiento de la cadena alimentaria. Los precios reales de los alimentos aumentaron más del $3 \%$ en el año 2008 provocando una disminución del poder adquisitivo y convirtiéndose en uno de los principales factores determinantes de la inflación global. En la UE, las prácticas comerciales desleales fueron por primera vez objeto de debate en el sector alimentario en 2009 , cuando los precios al consumo aumentaron a raíz de las subidas de los precios agrícolas ${ }^{33}$. El origen del auge de las leyes de competencia y la primera vez que se plantea el debate de las prácticas comerciales desleales confluyen en la crisis de los precios de los alimentos de 2008 .

\footnotetext{
${ }^{30} \mathrm{CNC}$, Informe sobre las relaciones..., op.cit., pág. I47.

${ }^{31}$ Se entiende por Mercas los Mercados Centrales de Abastecimiento, a propósito

http://www.sepi.es/default.aspx? $\mathrm{cmd}=0004 \&$ idContraste=\&IdContent=IIII3\&idLanguage=\&opcion=I $2 \&$ lang=

${ }^{32}$ «Coemfe critica a Agricultura por ignorarle en la Ley de Mejora de la Cadena Alimentaria», Revista Aral, 4.02.2016, (disponible en

http://www.revistaaral.com/es/notices/2016/02/coemfe-critica-a-agricultura-por-ignorarle-en-la-ley-demejora-de-la-cadena-alimentaria-7607ı.php\#.V9okok2LTIU;

fecha de consulta: 02.10.2016).

${ }^{33}$ Comisión Europea, Libro verde..., op. cit., pág. 3.
} 
La Decisión de la Comisión 2008/359/EC creó un Grupo de Alto Nivel (GAN) en abril de 2008 sobre la competitividad de la industria agroalimentaria y se aprobó la Comunicación de la Comisión 2009/59I en octubre de 2009 que pretendía mejorar el funcionamiento de la cadena alimentaria. En julio de 20 ıо se aprobó el Foro de Alto nivel sobre la mejora del funcionamiento de la cadena alimentaria a través de la Decisión de la Comisión 2010/C210/03. En 20II, la Plataforma de Expertos constituida dentro del Foro formuló una serie de principios y ejemplos de prácticas leales y desleales en las relaciones verticales de la cadena de suministro alimentario, a los que se adhirieron distintas organizaciones representativas de los diferentes intereses en toda la cadena europea de suministro alimentario ${ }^{34}$. En 2013 la Comisión publicó el Libro Verde sobre prácticas comerciales desleales en la cadena de suministro alimentario y no alimentario entre empresas en Europa. En julio de 2014 se aprobó la Comunicación de la Comisión $2014 / 472^{35}$ con objeto de hacer frente a las prácticas comerciales desleales entre empresas.

El Libro Verde consideraba que la existencia de normas nacionales fragmentadas respecto a la cadena alimentaria podía constituir un obstáculo para el abastecimiento y la distribución transfronteriza en el Mercado único ${ }^{36}$. Mejorar el funcionamiento de la cadena de suministro propiciaría una mejor integración económica y permitiría corregir ciertas deficiencias causadas por las prácticas comerciales desleales en el Mercado único y soslayar la fragmentación de las normas nacionales destinadas a luchar contra tales prácticas. Ello contribuiría al objetivo de la UE de convertirse en una economía más inteligente, sostenible e integradora dentro de la Estrategia europea para el crecimiento, Europa 2020. Además, se preveía que las divergencias entre las distintas legislaciones iban a aumentar consecuencia del comercio electrónico y de la globalización ${ }^{37}$.

El Libro Verde pretendía ser, precisamente, un documento de reflexión y debate con objeto de evitar esta dispersión. Asimismo, en septiembre de 2013 , se aprobó un código voluntario de buenas prácticas a nivel europeo promovido por varias asociaciones del sector como AIM, CELCAA, EUROCOMMERCE, FOODDRINKEUROPE, UGAL, EUROCOOP y ERRT. Se pretendía homogeneizar el tratamiento que se realizaba de las buenas prácticas en la Unión Europea. Llama la atención que estas asociaciones representan a grandes empresas alimentarias que están excluidas de la aplicación de la LCA en España.

La regulación de las relaciones de la cadena alimentaria para intentar frenar determinadas prácticas abusivas no es un fenómeno típicamente español. En efecto, diversos Estados Miembros (Alemania, Francia, República Checa, Portugal e Italia) han adoptado ya desde hace años diversos sistemas con el mismo objetivo y con mayor o menor éxito como confirma CREDA en el Informe sobre la aplicación de regulaciones de las prácticas comerciales en la cadena alimentaria en los países de la UE en abril de $20 \mathrm{6} 6$.

\footnotetext{
${ }^{34}$ Ibidem, pág. 4

${ }^{35}$ Comisión, Comunicación para hacer frente a las prácticas comerciales desleales en la cadena de suministro alimentario entre empresas, COM/20I4/0472 final, Estrasburgo, I5.7.20I4, págs. I-I5

${ }^{36}$ Ibidem, pág. 6.

${ }^{37}$ Comisión, Comunicación para hacer frente..., op.cit., pág. I3.
} 
En concreto, los países que tienen modelos similares al español son Alemania, Eslovaquia, Francia, Italia, Portugal, Reino Unido y República Checa. Cinco de ellos (Eslovaquia, Italia, Reino Unido y República Checa, además de España) ${ }^{3^{8}}$, cuentan con una normativa específica para el sector, mientras que los otros tres (Alemania, Francia y Portugal) disponen de leyes genéricas que se aplican a diferentes sectores económicos y no son exclusivas para el sector alimentario. Además, en Eslovaquia (Ministerio de Agricultura), República Checa (Oficina para la protección de la competencia UOHZ), Italia (Autoridad Garante de la competencia y del mercado AGCM) y Reino Unido (Groceries Code Adjudicator (GCA) existen organismos específicos del sector alimentario similares a la AICA española como autoridades de control ${ }^{39}$.

En enero de 2016 la Comisión emitió un informe ${ }^{40}$ en el que reconocía que se había producido un desarrollo legislativo importante en veinte Estados miembros acerca de la adopción de medidas contra las prácticas comerciales desleales. No obstante, consideraba que un enfoque regulador armonizado en todo el espacio europeo no añadiría ningún valor. Es decir, la Comisión consideraba que no era necesario armonizar toda la legislación diversa existente en los Estados miembros en unas directrices únicas para todos. Opinión de la Comisión, esta última, que suscita bastante recelo.

En España, como respuesta a la Comunicación de la Comisión 2009/59I que animaba a los Estados miembros a regular nacionalmente sus cadenas alimentarias, se aprobó la LCA. Esta ley surge con objeto de frenar las prácticas abusivas que se vienen produciendo en las relaciones comerciales entre los actores de la cadena y que ya se pusieron de manifiesto por la antigua Comisión Nacional de Competencia en su Informe de 2011 sobre las relaciones entre fabricantes $y$ distribuidores en el sector ${ }^{41}$. En las recomendaciones que realiza la CNC se abordan aspectos como la necesidad de formalizar los contratos por escrito, especificar los pagos, suprimir la regulación de la venta a pérdida del artículo i4 de la LORCOMIN con objeto de mantenerla dentro de la legislación de competencia ${ }^{42}$. Es decir, la propia CNC se anticipa al articulado de la LCA que materializará esas necesidades que ya previó la autoridad nacional de competencia constituida por la extinta CNC. La autoridad nacional de competencia se adelanta a las necesidades de la ley específica nacional.

El fin que pretende la CNC es favorecer un entorno competitivo y eficiente que maximice el bienestar de los consumidores finales. No obstante, hace hincapié en que se deben evitar la proliferación de diferentes regímenes regulatorios o códigos de conducta de las prácticas comerciales entre proveedores y distribuidores dentro del territorio nacional.

\footnotetext{
${ }^{38}$ CREDA y AICA, op.cit., pág. II.

${ }^{39}$ CREDA y AICA, op. cit., pág. 42.

${ }^{40}$ Comisión Europea, 20i6, Comunicación de la Comisión (COM 20I6/32). Informe de la Comisión al Parlamento Europeo y al Consejo sobre las prácticas comerciales desleales en la cadena de suministro alimentario entre empresas.

${ }^{4 \mathrm{~T}}$ CRESPO, D. et al., op.cit, pág. 2.

${ }^{42} \mathrm{CNC}$, Informe sobre las relaciones..., op.cit., págs. I47-I49.
} 
Insta a las Administraciones Públicas y a las asociaciones sectoriales a que sean consecuentes para evitar la proliferación de códigos de conducta ${ }^{43}$.

España se sitúa como país pionero en implantar una regulación específica para la cadena alimentaria y líder en la UE en la creación de una normativa propia para el sector. La normativa española ha servido como modelo a otros países de la UE que han establecido normas similares en sus respectivos Estados con el objetivo de lograr el equilibrio en las relaciones comerciales ${ }^{44}$. Esta ley española se aplica a todos los eslabones de la cadena alimentaria a diferencia de lo que ocurre en otros Estados miembros en donde la legislación sobre prácticas comerciales desleales se aplica solo a los últimos eslabones de la cadena alimentaria. Es el caso del Reino Unido, Eslovaquia y República Checa donde la normativa solo se aplica a determinados minoristas y a su relación directa con los proveedores ${ }^{45}$.

Por cadena alimentaria se entiende el conjunto de actividades que llevan a cabo los distintos operadores que intervienen en la producción, transformación $y$ distribución de alimentos o productos alimenticios, excluyendo las actividades de transporte y de la hostelería y la restauració $n^{46}$. Se considera que tanto la hostelería como la restauración revisten características muy distintas a las que se dan en el canal de la alimentación por lo que quedan excluidos de la aplicación de la presente ley los establecimientos minoristas como hoteles, restaurantes, bares y cafeterías ${ }^{47}$. Esta excepción tiene una enorme repercusión en España debido al amplio número de relaciones contractuales que se dan entre la distribución alimentaria y las empresas que se dedican a la hostelería y la restauración.

El artículo 2 establece que la LCA se aplicará a las relaciones comerciales que se produzcan entre los operadores que intervengan en la cadena alimentaria, desde la producción, pasando por la industria transformadora hasta la distribución. También quedan incluidas las operaciones comerciales que se realicen entre operadores de la cadena alimentaria en los procesos de envasado, transformación o acopio para su posterior comercialización, así como las compras de animales vivos, piensos y todas las materias primas e ingredientes utilizados para la alimentación animal. Por el contrario, no tienen la consideración de relaciones comerciales y por tanto quedan excluidas del ámbito de aplicación de la Ley las entregas de producto a cooperativas agrarias y otras entidades asociativas, por parte de los socios de las mismas, siempre que por previsión de los estatutos de la entidad correspondiente vengan obligados a la realización de dicha entrega ${ }^{48}$.

\footnotetext{
${ }^{43}$ Ibidem, pág. I49.

${ }^{44}$ MAGRAMA, «España se sitúa como líder europeo en regular las operaciones comerciales de la cadena alimentaria», Madrid, 7.06.2016, (disponible en

http://www.magrama.gob.es/es/prensa/noticias/-espa\%C3\%Bıa-se-sit\%C3\%BAa-como-1\%C3\%ADdereuropeo-en-regular-las-operaciones-comerciales-de-la-cadena-alimentaria-/tcm7-423630-16; fecha de consulta: Io.10.20I6).

${ }^{45}$ CREDA y AICA, op. cit., pág. 48.

${ }^{46}$ Artículo 5.a) de la LCA.

${ }^{47}$ Moya Latorre, A. et al., op. cit., págs. 663-665.

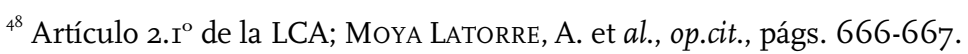


Llama la atención que esta legislación específica no se aplica a las grandes empresas de alimentación que han sido las grandes promotoras de la ley y son las encargadas de organizar la actual distribución moderna ${ }^{49}$. El artículo 2.3 a) especifica que dentro del ámbito material de la ley, uno de los operadores debe ser pyme y el otro no. De esta manera, quedan excluidas las relaciones entre las grandes empresas de alimentación y distribución. Es como si el volumen de negocio que generan si se pudiera regular por la legislación de competencia y, sin embargo, el negocio que generan las pymes y el sector primario fuera necesario reforzarlo a través de esta LCA porque sufren un abandono por parte de la legislación de competencia. Que las grandes industrias alimentarias queden excluidas de esta ley es, cuando menos, gravoso.

Y por último, quedan excluidas de esta ley específica las relaciones comerciales con los consumidores finales que se regulan en la Ley General para la Defensa de los Consumidores y Usuarios (LGDCU) y en otras leyes complementarias ${ }^{5 \circ}$, cuya competencia corresponde respectivamente a cada CCAA.

La LCA regula prácticas formales y prácticas materiales. Dentro de las formales destaca la formalización de contratos por escritos cuando se realice una compraventa y el importe de la transacción sea superior a 2500 y que además una de las partes pertenezca al sector primario y el otro no, o que una de las partes sea una pyme y el otro no o por último, que exista una relación de dependencia de uno a otro ${ }^{51}$. Muy importante es que se trate de transacciones cuya cuantía sea superior a los 2500 , no se haya efectuado un pago al contado (porque en tal caso no se aplica esta ley), que una de las dos partes de la transacción pertenezca al sector primario y que exista una relación de dependencia económica que lógicamente sitúa a la parte más débil en una clara situación de inferioridad. No obstante, la LCA solo impone la forma escrita para los contratos alimentarios que aparecen regulados en los artículos 8 a II, en el Capítulo I Título II ${ }^{52}$.

El fin último de esta práctica formal es dejar constancia de que se ha celebrado un contrato y evitar que se den situaciones en las que se vendan las cosechas sin precio, por ejemplo. Solo de esta manera se puede proteger a la parte débil de la relación contractual si la hubiera. Con la ausencia de contratos que permite la libertad contractual, resultaba muy difícil rastrear y perseguir los abusos que se producían en las compraventas de palabra. La Ley exige la formalización de contratos por escrito y un contenido mínimo obligatorio que incluya necesariamente la identidad de las partes, el objeto, el precio, las condiciones de pago, las condiciones de entrega, la duración del contrato y las causas de extinción si las hubiera, entre otros extremos ${ }^{53}$.

Respecto a las obligaciones materiales que regula la LCA destacan la prohibición de prácticas abusivas como son las modificaciones unilaterales en los contratos y los pagos no

\footnotetext{
${ }^{49}$ CRESPO, D. et al., op.cit., pág. 3 .

${ }^{50}$ Real Decreto Legislativo I/2007, de I6 de noviembre de 2007. BOE 30.II.2007.

${ }^{51}$ Artículo 3 de la LCA.

${ }^{52}$ Artículo $2.4^{\circ}$ y artículo 8 de la LCA.

${ }^{53}$ Artículo 9 de la LCA.
} 
previstos, el suministro de información comercial sensible ${ }^{54}$, la gestión de marcas ${ }^{55}$ y el incumplimiento de los plazos de pago ${ }^{56}$. Todas estas conductas podrían haberse perseguido por la Ley de competencia, Ley de competencia desleal, Ley de marcas, Ley contra la morosidad y Ley de publicidad. Pero parece que estas leyes no han perseguido los ilícitos que motivaron su aprobación.

Hasta la fecha, el Observatorio de la cadena alimentaria ha realizado estudios sobre los precios de la leche y del conejo, pero se echan en falta más estudios. Los artículos 22 a 26 establecen el régimen sancionador y la potestad sancionadora. A propósito de las sanciones, se debe tener en cuenta que si se ha iniciado un procedimiento judicial o un procedimiento administrativo de defensa de la competencia, primarán estos porque no se pueden enjuiciar los mismos hemos por dos vías distintas. Sorprende las escasas disposiciones materiales que tiene la LCA. La mayor parte de su contenido es de carácter institucional o procedimental.

No obstante, respecto al desarrollo del procedimiento establecido en la Ley, siguen sin regularse algunos aspectos. En primer lugar, no hay desarrollo reglamentario respecto a los contratos tipo que fomenta la Ley para integrarlos en la cadena alimentaria. Hasta el momento, la operativa es que los operadores envían el borrador del contrato a la AICA quien lo supervisa, lo modifica y con su visto bueno lo devuelve para que se pueda aplicar. Falta desarrollo reglamentario del sistema de arbitraje propuesto para resolver los conflictos que puedan darse dentro de los operadores de la cadena que se hayan adherido al CBPCA. Tampoco se ha desarrollado la Ley de Interprofesionales ni se ha dado contenido al sello que propuso el MAGRAMA para identificar a unos operadores frente a otros. Desde que entró en vigor la LCA se ha avanzado mucho pero aún quedan muchas cosas por hacer.

\section{Legislación de defensa de la competencia y la Ley de la cadena alimentaria}

La doctrina se pregunta por qué se aprueba una Ley de limitación de la autonomía de la voluntad, de control de comportamientos para una actividad concreta ¿No es suficiente el recurso a las normas de defensa de la competencia? Y ¿por qué no bastan? ¿Este defecto se aplica únicamente a este sector? Cuántos sectores, además de este, deberían ser regulados por el legislador de manera específica ${ }^{57}$.

La falta de transparencia del mercado, los desequilibrios en el poder de negociación y las prácticas contrarias a la competencia originan distorsiones del mercado que pueden

\footnotetext{
${ }^{54}$ Artículo I3 de la LCA.

${ }^{55}$ Artículo I4 de la LCA.

${ }^{56}$ El artículo $23.2^{\circ}$ de la Ley I2/2013 remite a la Disposición Adicional Primera de la Ley I5/2010 de 5 de julio, de modificación de la Ley 3/2004, de 29 de diciembre por la que se establecen medidas de lucha contra la morosidad en las operaciones comerciales (BOE 6.07.2010).

${ }^{57}$ Araujo, M. y Ferrer, E., «La Ley de medidas para mejorar el funcionamiento de la cadena alimentaria. Una visión desde el derecho de la competencia», La Ley, número 35, septiembre octubre 20I3, págs. I6-2I, especialmente pág. I6.
} 
repercutir negativamente en la competitividad de la cadena de suministro alimentario en su conjunto. Se hace patente la necesidad de analizar el funcionamiento de la cadena de suministro alimentario. Parece que existe una relación de causa-efecto entre las prácticas contrarias a la ley de competencia y el funcionamiento de la cadena alimentaria.

La Comisión consideró que las ofertas hechas a los consumidores no eran suficientemente leales en términos de gama de productos y precios y que los intermediarios, transformadores agroalimentarios y minoristas comprimían los márgenes de los productores agrícolas ${ }^{58}$ ¿Se debe seguir protegiendo a los agricultores y ganaderos con las ayudas de la PAC como lleva ocurriendo más de 50 años y, a la vez, permitir que se les asfixie con los márgenes comerciales en la cadena alimentaria? Es un sinsentido. Para Tomás Azcárate, el sector primario debe defender su posición en la cadena alimentaria. Cada vez tiene menos poder y menos peso y debería ser al revés.

La autoridad española de defensa de la competencia se ha pronunciado en diversas ocasiones sobre la LCA. En primer lugar, en diciembre de 20I2, en el IPN 84/12 Anteproyecto de Ley de medidas para mejorar el funcionamiento de la cadena alimentaria. En segundo lugar, en el Informe artículo 25 Tramitación proyecto de Ley de la Cadena Alimentaria y además, en el IPN/DP/0009/14 Proyecto de Real Decreto por el que se aprueba el Reglamento de la Ley 12/2013, de 2 agosto, de medidas para mejorar el funcionamiento de la cadena alimentaria en julio $2014^{59}$. Parece evidente que los informes que emite la autoridad de defensa de la competencia a nivel estatal no coinciden ni respaldan el funcionamiento, el procedimiento y el régimen sancionador de la LCA. Se observan tensiones por la compatibilidad (o no) de ambas legislaciones.

La CNMC cuestionó la inseguridad jurídica que la aprobación de la LCA podía generar y destacó la burocracia innecesaria y desproporcionada que iba a desplegarse para regular las relaciones comerciales entre los agentes de la cadena. Advirtió de los efectos negativos que podía tener para la competencia la introducción de la figura del mediador en la medida en que podía facilitar una coordinación anticompetitiva de los operadores a través de ciertas recomendaciones de precios, conducta prohibida por el artículo I de la LDC. No obstante, la CNMC se estaba adelantando mucho con la figura del mediador. En primer lugar porque aún no se ha creado ni desarrollado reglamentariamente (estaba previsto su desarrollo en los programas electorales de los partidos políticos) y en segundo lugar porque no necesariamente debe recaer su actuación en las recomendaciones de precios prohibidas por la legislación de competencia.

En el IPN 84/12 sobre el Anteproyecto de Ley de medidas para mejorar el funcionamiento de la cadena alimentaria, la autoridad de competencia consideraba que el régimen jurídico sancionador contemplado en la LCA se solaparía con los ya existentes en la legislación de competencia. Las relaciones contractuales mercantiles entre operadores

\footnotetext{
${ }^{58}$ Comisión Europea, Libro verde..., op.cit., pág. 4.

${ }_{59}$ CNMC, IPN/DP/o0o9/I4 Proyecto de Real Decreto por el que se aprueba el Reglamento de la Ley I2/20I3, de 2 agosto, de medidas para mejorar el funcionamiento de la cadena alimentaria, julio 20I4, Madrid, págs. I-II, especialmente pág. 4 .
} 
económicos se encontraban ya sujetas a la normativa de obligaciones y contratos, a la legislación de competencia desleal, a la legislación de propiedad industrial, y a la legislación de defensa de la competencia. Por lo tanto, la autoridad de competencia mostró su rechazo a la aprobación de un nuevo régimen sancionador en la LCA $^{60}$ porque podrían llegar a producirse solapamientos de todos estos regímenes.

El Proyecto de RD IPN/DP/ooog/I4 de la CNMC destacaba los riesgos de imparcialidad, independencia y objetividad que representaba la integración de la CNMC en el Observatorio de la cadena alimentaria, en la AICA y la voluntariedad en la adhesión al CBPCA. En el extinto Observatorio de los precios de los alimentos, la autoridad de competencia venía asistiendo voluntaria y estrictamente en calidad de observador, con voz pero sin voto. De esta forma, las decisiones y la independencia de criterio de dicha autoridad quedaban preservadas. La CNMC considera necesario, a efectos de salvaguardar su independencia y la aplicación eficaz de la normativa de defensa de la competencia, que su posible asistencia se realice únicamente como miembro observador u otra figura jurídica similar.

El Estatuto de la AICA, previsto en el Real Decreto 227/20I4, regula el Consejo Asesor de la Agencia señalando que es el órgano consultivo y de participación en el que se integran representantes de la Administración General del Estado, de las Comunidades Autónomas, de la producción y la transformación de los sectores alimentarios que estén interesados y de la distribución y los consumidores. Entre los sesenta miembros del Consejo Asesor habrá un representante de la CNMC. Los razonamientos que se esgrimen para la participación de un miembro de la CNMC es que éste se mantenga imparcial y objetivo para que no surjan incompatibilidades con el desempeño de su cargo. Asimismo, se matizan dos aspectos. En primer, en contra de lo que dispone el artículo $5.2^{\circ}$ de la Ley 3/2013 de 4 de junio, de creación de la Comisión Nacional de los Mercados y la Competencia $^{61}$, la CNMC sugiere que cuando asista al Consejo Asesor de la AICA no se le exija emitir ningún informe y, en segundo lugar, que se valore la actividad sancionadora de la propia Agencia porque podrían solaparse sus sanciones con otras impuestas por la autoridad de defensa de la competencia. La CNMC debería tener el estatus de miembro observador, con voz pero sin voto, en el Estatuto de la AICA.

Por su parte, la CNMC matiza que cuando se trate de la aprobación de normas que tengan implicaciones para la legislación de competencia, haciendo uso de las facultades que recoge el artículo $5.2^{\circ}$ de la Ley $3 / 2013$, de 4 de junio, de creación de la CNMC, sería oportuno que fuera preguntada y que, además, emitiera un informe previo al respecto. Está

${ }^{60}$ IPN 84/I2 sobre el Anteproyecto de Ley de Medidas para Mejorar el funcionamiento de la Cadena Alimentaria, Madrid, 20I2, pág. 8.

\footnotetext{
${ }^{61}$ Artículo $5.2^{\circ}$ de la Ley $3 / 2013$, de 4 de junio, de creación de la Comisión Nacional de los Mercados y la Competencia establece que la Comisión Nacional de los Mercados y la Competencia actuará como órgano consultivo sobre cuestiones relativas al mantenimiento de la competencia efectiva y buen funcionamiento de los mercados $y$ sectores económicos. En particular, podrá ser consultada por las Cámaras Legislativas, el Gobierno, los departamentos ministeriales, las Comunidades Autónomas, las Corporaciones locales, los Colegios Profesionales, las Cámaras de Comercio y las Organizaciones Empresariales y de Consumidores y Usuarios. En ejercicio de esta función, llevará a cabo las siguientes actuaciones: Informes...
} 
claro que respecto a algunas materias si está interesada en involucrarse y respecto a otras materias no.

El artículo 49. $\mathrm{I}^{\circ}$ de la LDC permite a cualquier persona física o jurídica, interesada o no, poder formular denuncia de las conductas reguladas en esta Ley. La doctrina considera que se recoge implícitamente la conexión entre la AICA y la CNMC.

La CNMC, por su parte, critica que las ayudas y las subvenciones del Ministerio vayan a dar prioridad a aquellos solicitantes adheridos al CBPCA porque las ayudas deben dirigirse a corregir los fallos de mercado y no a otro motivo. El desequilibrio o la asimetría entre las partes contratantes no es una anomalía o un fallo del mercado sino una característica inherente a él. Los operadores del mercado deben dar respuesta a este desequilibrio desde un punto de vista dinámico, adaptando su producción y mejorando su eficiencia para ser más competitivos mientras fomentan la innovación. Si se otorgan subvenciones a aquellos que se adhieren al CBPCA, ello puede influir en el carácter voluntario de la adhesión ${ }^{62}$.

La LCA ha sido consecuencia del debate que se originó con el Informe sobre las relaciones entre fabricantes y distribuidores en el sector alimentario publicado en octubre de 20 II por la CNC. Se podría considerar que el germen de esta ley que regula la cadena alimentaria proviene de los organismos de defensa de la competencia.

Esta ley específica busca completar el ordenamiento de defensa de la competencia. El artículo 7 de la LCA dispone que el contenido de las relaciones reguladas por la presente Ley, así como la aplicabilidad de los principios rectores en la ejecución e interpretación de tales relaciones, quedará sometido a la normativa de defensa de la competencia, sin perjuicio de lo dispuesto en la normativa comunitaria. Sin embargo, su aplicación se encomienda a órganos administrativas y no a la autoridad de competencia.

ARAUJO y FERRER consideran que la legislación de la cadena alimentaria representa unas normas de intervención extraordinaria a favor de un sector de actividad. Los poderes públicos defienden la parte más débil de la relación. Se trata de disposiciones de defensa de la competencia en sentido material pero divergen en sus técnicas de aplicación ${ }^{63}$. Su aplicación se encomienda a organismos específicos a los que se habilita a definir una política de forma análoga a lo que contemplan las normas de defensa de la competencia sin perjuicio del control que lleven a cabo los tribunales de justicia. Existen similitudes en las facultades conferidas a los inspectores de la AICA y a las autoridades de competencia. La diferencia más importante estriba en la cuantía de las multas porque son considerablemente inferiores las que puede imponer la AICA frente a las que pueden imponer las autoridades de competencia. Las multas que imponen los órganos de

\footnotetext{
${ }^{62}$ CNMC, IPN/DP/Ooo9/I4 Proyecto de Real Decreto por el que se aprueba el Reglamento de la Ley I2/20I3, de 2 agosto, de medidas para mejorar el funcionamiento de la cadena alimentaria, Madrid, julio 20I4, págs. I-II, especialmente pág. IO.

${ }^{63}$ ARAujo, M. et al., op.cit., pág. I8.
} 
competencia pueden llegar a representar el ı०\% del volumen del negocio de la compañía en un determinado mercado ${ }^{64}$.

El contenido de la LCA es fundamentalmente institucional o procedimental, tiene pocos artículos que desarrollen el contenido material de la ley. Los artículos i2 a i4 prohíben conductas concretas y son complementarias al ordenamiento de defensa de la competencia en el sector alimentario ${ }^{65}$. Establecen un nexo entre ambas legislaciones y remiten a la fundacional que en este caso es la normativa de competencia.

El artículo I2.I ${ }^{\circ}$ LCA prohíbe que se pueda modificar unilateralmente cualquier contrato, igual que lo establece el artículo I256 del Código civil ${ }^{66}$. Si hubiera un abuso de una de las partes, se produciría la nulidad del contrato conforme lo dispuesto en el artículo $6.3^{\circ}$ del Código civil. El fin último de esta regulación es evitar que una parte doblegue a la otra. El artículo I2.2 $2^{\circ}$ LCA prohíbe la realización de pagos adicionales salvo aquellos que fueran necesarios para referenciar un producto o para financiar parcialmente una promoción.

El artículo I3 LCA regula el suministro de información comercial sensible. Para ello es necesario que exista un contrato por escrito, una información adecuada al objeto del contrato y que sea impuesta por una parte a la otra. El artículo I3.I ${ }^{\circ}$ hace una remisión explícita a las normas de defensa de la competencia.

El artículo I4 de la LCA regula la gestión de las marcas propias y de terceros de los productos alimentarios evitando las prácticas contrarias a la libre competencia, la competencia desleal o la general de publicidad. La expansión de las marcas propias de los distribuidores ha convertido a algunos comerciantes en competidores directos de sus proveedores $^{67}$. Se prohíbe el aprovechamiento indebido de la iniciativa empresarial ajena y cualquier acción que pueda generar riesgo de asociación o confusión entre unos productos y otros como puede ser el uso de envases. La LCA alude expresamente a otras normas que ya contemplan este tipo de prácticas como la Ley 15/2007 de Defensa de la Competencia, Ley 3/I99I de Competencia Desleal, Ley 34/I988 General de Publicidad y Ley I7/200I de Marcas. Este artículo I4 no sería necesario si se aplicaran adecuadamente las leyes que se citan. Lo relevante en este caso es la posibilidad de que estas normas se apliquen por una institución especializada en el control de las prácticas comerciales a lo largo de la cadena alimentaria, con poderes de investigación propios y con capacidad sancionadora propia ${ }^{68}$.

El Libro verde aclaró que el derecho de la competencia y las prácticas comerciales desleales no son lo mismo. Mientras que el derecho de la competencia vela por la libre competencia en el mercado, las prácticas comerciales desleales se refieren a las condiciones

\footnotetext{
${ }^{64}$ Lillo Alvarez, C., op.cit., pág. 2.

${ }^{65}$ ARAujo, M. et al., op.cit., pág. I7.

${ }^{66}$ Artículo I256 del Código civil establece que «la validez y el cumplimiento de los contratos no pueden dejarse al arbitrio de uno de los contratantes».

${ }^{67}$ Comisión Europea, Libro verde..., op.cit., pág. 3.

${ }^{68}$ CRESPO, D. et al., op. cit., pág. 7 .
} 
contractuales con independencia de que la competencia exista o no en el mercado ${ }^{69}$. El Derecho de la competencia de la UE pretende contribuir a la construcción y a la preservación del Mercado único y a la mejora del bienestar de los consumidores. Su objetivo es establecer condiciones en las que el mercado pueda funcionar adecuadamente y no se centra en la lealtad de las relaciones empresariales individuales, a menos que estas conlleven una disfunción del mercado debido a la existencia de un poder de mercado. Así pues, el Derecho de la competencia de la UE podría aportar una respuesta a ciertas prácticas comerciales desleales, pero no a todas ${ }^{70}$.

El considerando nueve del Reglamento I/2003 del Consejo de I 6 de diciembre de 2002 (DOCE 4.OI.2003) relativo a la aplicación de las normas sobre competencia previstas en los artículos 8I y 82 del Tratado (hoy artículos io y IO2 del TFUE) traza explícitamente una línea divisoria entre el Derecho de la competencia y la legislación sobre prácticas comerciales desleales. Establece que los artículos 8I y 82 tienen por objeto la protección de la competencia en el mercado. Ello no impedirá que los Estados miembros apliquen en sus territorios la legislación nacional que proteja otros intereses legítimos, siempre que esa legislación sea compatible con los principios generales y las demás disposiciones del Derecho europeo.

La LCA en España tiene como finalidad mejorar el funcionamiento y la vertebración de la cadena alimentaria de manera que aumente la eficacia y la competitividad del sector agroalimentario español y se reduzca el desequilibrio en las relaciones comerciales entre los diferentes operadores de la cadena de valor, en el marco de una competencia justa que redunde en beneficio no sólo del sector, sino también de los consumidores.

Lo que se pretende con la aprobación de la LCA es introducir cambios en cómo funcionan determinadas relaciones privadas mediante una intervención de los poderes públicos $^{71}$. Se observa que existen fallos en el funcionamiento o cierre de esas relaciones privadas y se piensa en la intervención pública con objeto de minimizarlos. Se parte de la constatación de la insuficiencia del mercado como instrumento que por sí mismo asegure la eficacia y competitividad del sector agroalimentario ${ }^{72}$. Existe la presunción de que el mercado es imperfecto y que necesita ciertos mecanismos para optimizar su rendimiento.

La doctrina parece tenerlo claro. Si la asimetría que existe en el mercado consistente en reducir el desequilibrio en las relaciones comerciales entre los diferentes operadores de la cadena de valor hubiera sido la única razón de peso para aprobar la LCA, se hubiera optado por mejorar o desarrollar las normas generales de protección de la parte débil contenidas en la Ley de competencia desleal y en la Ley de defensa de la competencia para las situaciones de dependencia económica. Pero esta no es la única razón. El legislador parte de la situación específica de un sector concreto, el sector alimentario, para el que

\footnotetext{
${ }^{69}$ Comisión Europea, Libro verde..., op.cit., pág. I2.

70 Ibidem, pág. I4.

${ }^{71}$ ArAujo, M., et al., op.cit., pág. 20.

${ }^{72}$ Ibidem, pág. I9.
} 
considera insuficientes las normas generales ${ }^{73}$. El legislador ve necesario profundizar en el sector alimentario porque considera que no está suficientemente protegido con las actuales leyes en vigor.

Se puede afirmar que existe una estrecha relación entre la Ley de la cadena alimentaria y las normas de defensa de la competencia. La Ley es el resultado de un debate producido en el seno de las autoridades de competencia ${ }^{74}$. Además, la rúbrica del Título II y del Capítulo II en donde se insertan las disposiciones materiales de la ley (artículos I2 a I4 de la LCA) hace referencia directa a prácticas comerciales abusivas, concepto cercano al ordenamiento de la competencia.

Como destaca el Preámbulo de la LCA, su finalidad es mejorar el funcionamiento y la vertebración de la cadena alimentaria.....en el marco de una competencia justa que redunde en beneficio no sólo del sector, sino también de los consumidores. La Ley de competencia también tiene como fin último la protección de los consumidores, así que estas dos leyes tienen, además, como denominador común la protección del consumidor ${ }^{75}$.

Se podría concluir que las disposiciones de la LCA buscan completar el ordenamiento de defensa de la competencia cuya aplicabilidad general se recoge en el artículo 7 de la Ley. Este complemento se realiza a través de mandatos que son normas de defensa de la competencia en sentido material, pero que difieren de las mismas en su aplicación. La normativa especial busca apoyar a una parte, considerada débil, en su relación con otra, que se entiende fuerte, algo que inicialmente no pretendía el ordenamiento $^{76}$. En este caso, la diferencia sustancial estriba en el planteamiento intrínseco de la debilidad de una de las partes de la relación contractual en la cadena alimentaria frente a la otra.

Esta situación de abuso de dependencia económica también aparece explícitamente regulado en el artículo ${ }_{1} 6.2^{\circ}$ de la Ley de Competencia Desleal. Se reputa desleal la explotación por parte de una empresa de la situación de dependencia económica en que puedan encontrarse sus empresas clientes o proveedores que no dispongan de alternativa equivalente para el ejercicio de su actividad. Esta situación se presumirá cuando un proveedor, además de los descuentos o condiciones habituales, deba conceder a su cliente de forma regular otras ventajas adicionales que no se conceden a compradores similares. La Ley de competencia desleal está omnipresente en la LCA. Los artículos I2, I3 y I4 de la LCA remiten a los artículos Io, in y I6 de la LCD. El conflicto se resuelve según cuál haya sido la autoridad que haya empezado a conocer del asunto. Si ha empezado a conocer de los hechos la autoridad de competencia,

\footnotetext{
${ }^{73}$ Araujo, M., et al., op.cit., pág. 20.

${ }^{74} \mathrm{CNC}$, Informe sobre las relaciones entre fabricantes $y$ distribuidores en el sector alimentario, octubre de 20II; Red de Competencia Europea, Report on competition law enforcement and market monitoring activities by European competition authorities in the food sector, 2012; Libro Verde sobre prácticas comerciales desleales en la cadena de suministro alimentario $y$ no alimentario entre empresas en Europa, 2013 y Dictamen del CESE sobre relaciones comerciales entre la gran distribución Y proveedores de productos alimentarios, DOUE Ci33 de 9 de mayo de 2013.

${ }^{75}$ Araujo, M. et al., op.cit., pág. I7.

${ }^{76}$ Ibidem, pág. 2I.
} 
será competente ésta. Si quien conoce en primer lugar es la AICA, determinará si continúa ella con el procedimiento o lo remite a la ley de competencia desleal.

Parece demostrarse que la Ley de la Cadena Alimentaria no suple la normativa de defensa de la competencia sino que la complementa ${ }^{7}$, ya que, mientras que la LCA pretende impedir prácticas desleales que guardan relación con los desequilibrios en el poder de negociación de las partes de una relación comercial, la ley de competencia persigue prácticas contrarias a la competencia relativas a la situación de poder en el mercado $^{78}$. El texto legal, algo ambiguo en algunos de sus artículos, debe interpretarse adecuadamente para limitar los abusos de mercado por parte de los operadores de la cadena que ocupan una posición dominante ${ }^{79}$.

El legislador ha considerado adecuado encomendar su aplicación a organismos e instituciones administrativas especiales y no a la autoridad de competencia. Esta decisión responde a la identificación del sector alimentario como un área en la que deben aplicarse reglas específicas de competencia, en el sentido material de la expresión, pero partiendo de una distinta técnica de aplicación.

La aprobación y entrada en vigor de la Ley de la cadena alimentaria representa un nuevo escenario para un sector que lleva mucho tiempo reclamando normas que atiendan a sus necesidades específicas, sin encontrar respuestas adecuadas en las disposiciones tradicionales de defensa de la competencia ni en ninguna otra. La nueva Ley abre un proceso de definición de un nuevo contenido para el sector agroalimentario que se va concretando día a día.

\section{Conclusiones}

Desecho la hipótesis que defendí al principio porque si bien es verdad que la Ley I2/20I3 se mueve ambiguamente en materia de derecho de la competencia (artículo $7 \mathrm{y}$ artículos I2 a I4 de la LCA), acaba regulando aspectos que no están debidamente tratados y desarrollados en la legislación de competencia. Esto hace que la LCA sea una legislación específica del sector agroalimentario e importante, en palabras de Araujo y Ferrer ${ }^{8 \circ}$. El sector agrario puede estar satisfecho ${ }^{81}$ porque hacía tiempo que reclamaba a las autoridades europeas ciertos cambios por abusos reiterados en sus relaciones comerciales, sin conseguirlo. Y con esta ley se ha dado respuesta a sus voces.

\footnotetext{
${ }^{77}$ Araujo, M. et al., op.cit., págs. I7 y 2 I.

${ }_{78}$ «El propósito de la Ley de la cadena alimentaria», Revista Inforetail, I0.04.20I4, (disponible en http://www.revistainforetail.com/noticiadet/el-proposito-de-la-ley-de-la-cadenaalimentaria/fbara23963052b6b27efi65e223 dfacc; fecha de consulta: 30.07.2016).

79 «España busca el equilibrio en la cadena alimentaria», Expansión, Madrid, I.07.2013, (disponible en http://www.promarca-spain.com/pdf/expansion_or.07.I3.pdf; fecha de consulta: 30.07.2016)

${ }^{80}$ ARAUjo, M. et al., op.cit., pág. 2I.

${ }^{81}$ COMPES Lopez, R., op.cit., pág. 238.
} 
Es decir, aunque la actual legislación nacional en competencia (Ley defensa de la competencia y Ley de competencia desleal) hubiera hecho su trabajo, hubiera sido necesaria una legislación específica que profundizara más en los aspectos que afectan y conciernen al sector agrario español porque se ha demostrado que la legislación nacional en competencia era insuficiente para regular todas las situaciones injustas y abusivas que se están produciendo en la cadena alimentaria y a las que la actual legislación en competencia no llegaba.

La LCA aparece como una ley complementaria a la legislación de competencia que deberá, con el tiempo, ir encontrando su lugar y desarrollándose como es debido. Aún está pendiente de completarse el Código de Buenas prácticas comerciales en la cadena, de desarrollar reglamentariamente el sistema de arbitraje previsto para la resolución de conflictos y el sello que identifique a aquellos operadores que se adhieran al Código de Buenas Prácticas.

En mi opinión, sería conveniente establecer unas directrices comunes para coordinar las actuaciones con las distintas CCAA. Asimismo, si la AICA designara órganos autonómicos a los cuales se pudieran dirigir los particulares para tramitar denuncias o abrir expedientes, los procedimientos serían mucho más ágiles. Por otro lado, se echan en falta más informes sectoriales. Pero se está avanzando, poco a poco.

Los inspectores de los organismos de defensa de la competencia (europeos, nacionales y autonómicos cuando los haya) tienen las mismas competencias que los inspectores de la AICA. Pueden entrar en los locales, realizar inspecciones, solicitar documentos y precintar instalaciones. Observo una diferencia fundamental. Mientras que los inspectores de la AICA están centralizados en Madrid y se desplazan para trabajar en distintas regiones españolas, en el caso de los organismos de defensa de la competencia, los inspectores pueden estar ubicados en el territorio autonómico objeto de estudio porque se haya establecido así en su Estatuto de Autonomía. Depende de cada región y cada caso. Eso representa una proximidad geográfica importante a la realidad y por lo tanto, a la infracción que se comete y a los autores de la misma.

La LCA y la legislación de competencia protegen bienes jurídicos distintos. Por un lado, la LCA persigue las conductas comerciales desleales con independencia de que exista o no libre competencia en el mercado. Por su parte, la legislación de competencia promueve el libre mercado y la libre competencia, incluso justifica alguna infracción a la libre competencia si hay un interés mayor que lo justifique y siempre que redunde en beneficio del propio consumidor. $\mathrm{O}$ al menos, cuando el consumidor obtenga algún beneficio económico proporcional al beneficio que ha reportado la conducta infractora.

Con la LCA se han desarrollado procedimentalmente ciertos aspectos. La autoridad encargada de vigilar, sancionar y controlar es una autoridad administrativa especial, no es un organismo de defensa de la competencia. Las sanciones son notablemente inferiores a las que impone la legislación de competencia. Y todo ello sin perjuicio de que se desvíen ciertos temas a las respectivas autoridades de competencia y a las autoridades jurisdiccionales cuando corresponda. Estas autoridades administrativas habrán de construir 
un sistema homogéneo y previsible proporcionando seguridad a los operadores económicos respecto de los comportamientos admisibles y los no aceptables. La UE promueve los códigos de autorregulación nacionales porque considera que los Estados son mejores conocedores de la realidad que atraviesan sus países.

Para erradicar la mala praxis que lleva arraigada desde hace muchos años en las compraventas de productos agrarios será crucial el papel que desempeñe la Administración. Es ella la que más puede presionar con las inspecciones y las multas que imponga para que el día a día cambie con objeto de que los diferentes eslabones de la cadena alimentaria hagan un esfuerzo (impuesto) por ajustarse a la Ley que les obliga a firmar contratos antes de la entrega de la producción o la cosecha con un contenido mínimo obligatorio y a guardar toda la documentación durante dos años.

Sería oportuno que actuaran complementariamente los órganos creados por la LCA y los órganos de defensa y promoción de la libre competencia. No obstante, se observa que operativamente existe cierta rivalidad entre ambos y se teme que la AICA deje a la luz deficiencias de los actuales organismos de defensa de la competencia o ponga en evidencia una falta de adaptación a esta Ley especial por parte de la Ley de competencia desleal, la Ley de defensa de la competencia y la legislación en materia de contratos y de propiedad industrial. Hay que confiar que no sea así. 